\title{
First Results on the Epoch of Reionization from First Light with SARAS 2
}

\author{
Saurabh Singh ${ }^{1,4}\left(\mathbb{1}\right.$, Ravi Subrahmanyan ${ }^{1}$ (i) , N. Udaya Shankar ${ }^{1}$ (i), Mayuri Sathyanarayana Rao ${ }^{1}$ (i), Anastasia Fialkov ${ }^{2}$ (1), \\ Aviad Cohen $^{3}$, Rennan Barkana ${ }^{3}$, B. S. Girish ${ }^{1}$, A. Raghunathan ${ }^{1}$ (D), R. Somashekar ${ }^{1}$, and K. S. Srivani ${ }^{1}$ \\ ${ }_{1}^{1}$ Raman Research Institute, C V Raman Avenue, Sadashivanagar, Bangalore 560080, India; saurabhs@ @rri.res.in \\ ${ }^{2}$ Harvard-Smithsonian Center for Astrophysics, Institute for Theory and Computation, 60 Garden Street, Cambridge, MA 02138, USA \\ ${ }^{3}$ Raymond and Beverly Sackler School of Physics and Astronomy, Tel Aviv University, Tel Aviv 69978, Israel \\ Received 2017 March 20; revised 2017 July 19; accepted 2017 July 31; published 2017 August 14
}

\begin{abstract}
Long-wavelength spectral distortions in the cosmic microwave background arising from the $21 \mathrm{~cm}$ transition in neutral hydrogen are a key probe of the Cosmic Dawn and the Epoch of Reionization. These features may reveal the nature of the first stars and ultra-faint galaxies that transformed the spin temperature and ionization state of the primordial gas. SARAS 2 is a spectral radiometer purposely designed for the precision measurement of these monopole or all-sky global $21 \mathrm{~cm}$ spectral distortions. We use $63 \mathrm{hr}$ nighttime observations of the radio background in the frequency band 110-200 MHz, with the radiometer deployed at the Timbaktu Collective in Southern India, to derive likelihoods for plausible redshifted $21 \mathrm{~cm}$ signals predicted by theoretical models. First light with SARAS 2 disfavors the class of models that feature weak X-ray heating (with $f_{X} \leqslant 0.1$ ) and rapid reionization (with peak $\frac{d T_{b}}{d z} \geqslant 120 \mathrm{mK}$ per unit redshift interval).
\end{abstract}

Key words: cosmic background radiation - cosmology: observations - dark ages, reionization, first stars methods: observational

\section{Introduction}

The Epoch of Reionization (EoR), beginning with first light from the first stars and ultra-faint galaxies and ending with the almost complete reionization of the primordial gas, marks an important period in the cosmic evolution of baryons (Barkana \& Loeb 2001; Ciardi \& Ferrara 2005; Zaroubi 2013; Barkana 2016; Haiman 2016; McQuinn 2016). There is considerable uncertainty and limited observational constraints on the astrophysical evolution in this period, including the nature of the first sources of light, and the thermal and ionization state of the intergalactic medium (IGM).

Current observational constraints on the EoR are either indirect or integrated in time. They include the Gunn-Peterson trough toward high-redshift quasi-stellar objects (QSOs; Fan et al. 2006; McGreer et al. 2015), which places the end of reionization at redshift $z \sim 6$; the evolution in the luminosity function of Ly $\alpha$ galaxies, which indicates an ionization fraction of $0.4-0.6$ at $z \sim 7$ (Zheng et al. 2017); detection of the EoR signature in the cosmic microwave background anisotropies, placing the average redshift of reionization $z_{r}$ between 7.8 and 8.8 (Hinshaw et al. 2013; Planck Collaboration et al. 2016); and upper limits on the kinematic Sunyaev-Zeldovich effect, limiting the extent of the EoR to $\Delta z_{r}<2.8$ (Planck Collaboration et al. 2016).

On the other hand, the redshifted $21 \mathrm{~cm}$ line from neutral hydrogen is a direct probe of the state of the gas in the EoR. Wouthuysen-Field (Wouthuysen 1952; Field 1958) coupling of the spin to the kinetic temperature via Ly $\alpha$ photons, gas heating via X-rays, and reionization via ultraviolet radiation generate spatial and temporal fluctuations in the $21 \mathrm{~cm}$ signal, all of which result in a redshifted $21 \mathrm{~cm}$ power spectrum with a monopole or all-sky global component that traces the mean cosmological evolution (Madau et al. 1997). Although a direct detection of the $21 \mathrm{~cm}$ signal continues to be elusive, the Precision Array for

\footnotetext{
4 Joint Astronomy Program, Indian Institute of Science, Bangalore 560012, India.
}

Probing the Epoch of Reionization (PAPER) radio interferometer derived lower limits in the range 5-10 K on the IGM temperature at $z=8.4$ based on upper limits to the power spectrum of $21 \mathrm{~cm}$ spatial fluctuations at that epoch; the derived limit depends on the assumed ionization fraction (Pober et al. 2015). Recently, additional upper limits on the $21 \mathrm{~cm}$ power spectrum have been reported by Murchison Widefield Array (MWA; Beardsley et al. 2016), and Low-Frequency Array (LOFAR; Patil et al. 2017).

While these experiments, as well as Hydrogen Epoch of Reionization Array (HERA) and Square Kilometre Array-Low (SKA-Low), work toward the detection of the $21 \mathrm{~cm}$ power spectrum, detection of the global $21 \mathrm{~cm}$ signal from the EoR could well prepare the way with useful constraints on the mean evolution (Furlanetto 2006; Furlanetto et al. 2006; Pritchard \& Loeb 2012), given that a single-element radiometer suffices for a detection and, under ideal conditions, the time required for achieving useful sensitivity is only a few minutes (Shaver et al. 1999; Sathyanarayana Rao et al. 2017b). The present uncertainty in the astrophysical parameters during the EoR allows for various possible global $21 \mathrm{~cm}$ signals. Hence, well-calibrated, wide-band radiometer measurements could pin down the underlying astrophysics (Morandi \& Barkana 2012; Mirocha et al. 2013; Cohen et al. 2016; Harker et al. 2016).

A non-detection by the Experiment to Detect the Global EoR Signature (EDGES) experiment, which targeted the global signal, placed a lower limit on the extent of reionization of $\Delta z_{r}>0.06$ (Bowman \& Rogers 2010). Analysis of such measurements up to $z \sim 12-15$ used to rely on theoretical predictions (Madau et al. 1997; Furlanetto 2006) that reionization occurred in the "saturated heating" limit, in which cosmic heating had occurred earlier and the IGM temperature no longer affected the $21 \mathrm{~cm}$ signal. However, Fialkov et al. (2014) showed that late heating, in which reionization features strong $21 \mathrm{~cm}$ absorption due to a still-cold IGM, is quite plausible, opening up a wide variety of possible $21 \mathrm{~cm}$ signals. Extrapolations of recent observations to high redshift also 
support such scenarios (Madau \& Fragos 2017; Mirocha et al. 2017).

A number of experiments are underway to detect the global $21 \mathrm{~cm}$ signal, including EDGES 2 (Monsalve et al. 2017), Large Aperture Experiment to Detect the Dark Ages (LEDA; Bernardi et al. 2016), Broadband Instrument for Global HydrOgen ReioNisation Signal (BIGHORNS; Sokolowski et al. 2015a), and Sonda Cosmológica de las Islas para la Detección de Hidrógeno Neutro (SCI-HI; Voytek et al. 2014). Attaining the necessary sensitivity to plausible signals is a formidable challenge: the cosmological signal needs to be discerned in the presence of radio frequency interference (RFI), instrumental systematics (Liu et al. 2013), ionospheric effects (Vedantham et al. 2014; Sokolowski et al. 2015b) and Galactic and extra-Galactic foregrounds, which can be 5-6 orders of magnitude brighter than the signal (Liu \& Tegmark 2012; Harker 2015). Fortunately, the foregrounds have been shown to be spectrally smooth to $\mathrm{mK}$ levels in the frequency range of $40-200 \mathrm{MHz}$ and can be modeled by smooth functions (Sathyanarayana Rao et al. 2017b). Similarly, the ionospheric effects-absorption, emission, refraction and the stochastic error due to temporal variations in total electron content (TEC) - result in spectrally smooth components (Vedantham et al. 2014; Sokolowski et al. 2015b) that may be subsumed by a smooth modeling of the foreground. However, the level of systematics is critically dependent on the radiometer design and calibration scheme, as well as data modeling strategies (Patra et al. 2013; Bernardi et al. 2015; Monsalve et al. 2017).

The Shaped Antenna measurement of the background RAdio Spectrum 2 (SARAS 2) is a spectral radiometer that aims to detect the global redshifted $21 \mathrm{~cm}$ signal from the EoR over 40-230 MHz. Below, we describe its design philosophy, calibration methodology, algorithms developed for RFI excision, and modeling of the foregrounds and instrumental systematics. We present results from first light upon deploying the system at a relatively radio-quiet site at the Timbaktu Collective in Southern India.

\section{SARAS 2 Spectral Radiometer}

SARAS 2 has a wide-band, wide-field monopole antenna deployed on open, level ground with receiver electronics enclosed in a unit below the antenna and below ground. The receiver is a correlation spectrometer in that the antenna signal is first split into two, then amplified separately in two parallel signal paths. The analog signals are transmitted on optical fiber to a signal processing unit located $100 \mathrm{~m}$ away, which is followed by a digital spectrometer that spectrally decomposes the signals, computes the complex cross-correlation between the signals, and records the spectra. The entire system operates on batteries and can be deployed at remote radio-quiet sites.

\subsection{The Antenna}

The SARAS 2 antenna is a sphere-disk monopole antenna (see Figure 1) in which a circular aluminum disk on the ground is one element and a sphere atop an inverted cone forms the second element; the sphere and cone are smoothly conjoined and the cone surface meets the sphere tangentially. The edge of a small circular hole at the center of the disk continues down as the outer conductor of a coaxial cable, with a central conductor that connects to the apex of the inverted cone. The antenna smoothly transforms into an unbalanced transmission line that

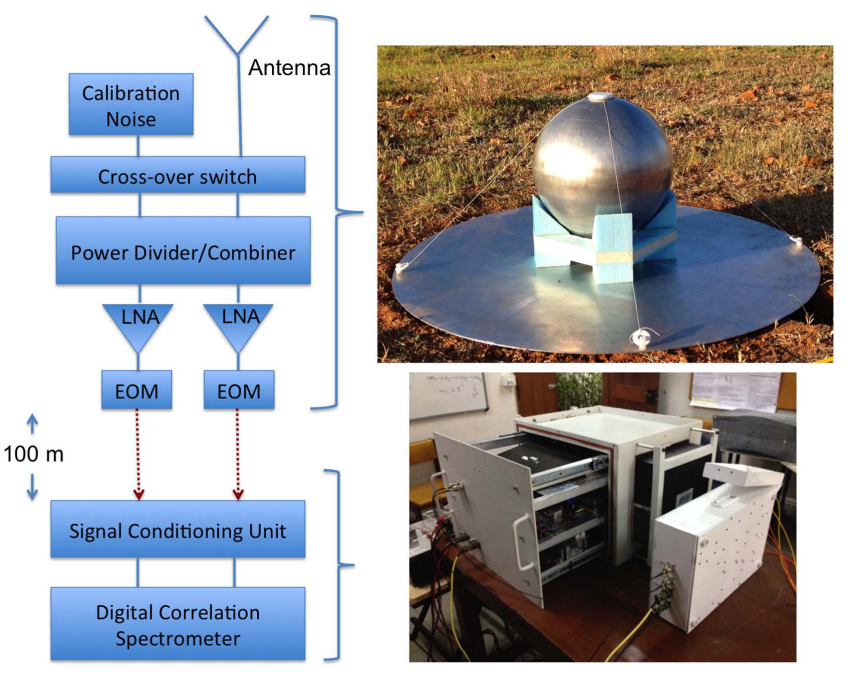

Figure 1. SARAS 2: in the schematic, LNA refers to low-noise amplifiers, while EOM are electro-optical modulators. The upper right image shows the sphere-disk monopole, with the sphere supported using styrofoam, cotton strings, and Teflon fasteners. The lower right image shows the spectrometer.

connects to the receiver below, thus avoiding any balun or impedance transformer that could introduce frequency-dependent resistive losses, which would be difficult to characterize to the required accuracy.

The antenna is electrically small with its spherical radiating element, of diameter $0.292 \mathrm{~m}$, less than $\lambda / 4$ at the highest frequency. Further, the disk radius is $0.435 \mathrm{~m}$, guaranteeing that the internal reflection of currents from the edge can only create sinusoids of period about $350 \mathrm{MHz}$ in the frequency-domain characteristics. The electrically small dimensions ensure that the entire observing band is within the first resonance, which is at $260 \mathrm{MHz}$. The structure has a simplistic design, defined by a minimal number of parameters, with smooth characteristics.

The antenna beam is omnidirectional, with nulls toward the horizon and zenith, with a peak at $30^{\circ}$ elevation and half-power beam width of $45^{\circ}$. Frequency independence of the beam is critical for this experiment in order to avoid the coupling of sky structure with spectral features. The electrically small dimensions ensure frequency independence for the antenna beam, and we have confirmed this property by range measurements and electromagnetic simulations.

A radiation efficiency $\eta_{r}(\nu)$ defines the frequency-dependent coupling of the beam-weighted sky temperature $T_{\text {sky }}(\nu)$ to the antenna. Owing to impedance mismatch between the antenna and transmission line, only a fraction of this power-defined by a reflection efficiency $\eta_{c}(\nu)$-arrives at the receiver. The total efficiency $\eta_{t}=\eta_{r} \times \eta_{c}$ determines the received antenna temperature:

$$
T_{a}(\nu)=\eta_{r}(\nu) \eta_{c}(\nu) T_{\text {sky }}(\nu) .
$$

Internal receiver noise appears as an additive contaminant in measured spectra, and internal reflections of the receiver noise at the antenna terminals result in spectral shapes for this contaminant, with the shape dependent on the antenna reflection coefficient $\Gamma_{c}(\nu)$, which is related to $\eta_{c}(\nu)$ as

$$
\eta_{c}(\nu)=1-\left|\Gamma_{c}(\nu)\right|^{2} .
$$

Thus, if $\Gamma_{c}$ has any low-level embedded ripples, then both foregrounds and receiver noise contributions in measured spectra would have a non-smooth structure. Therefore, 


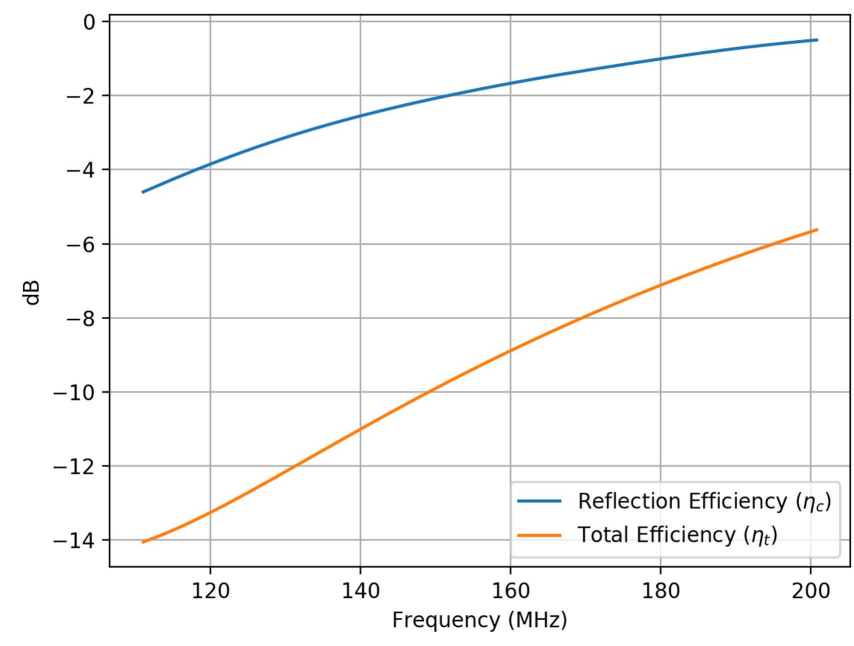

Figure 2. SARAS 2 antenna efficiencies vs. frequency.

designing $\Gamma_{c}$ to be spectrally smooth is critical for the detection of the EoR global signal. Mathematically, we require $\Gamma_{c}$ to be maximally smooth (Sathyanarayana Rao et al. 2015). As discussed above, the shape and dimensions of the antenna are chosen in order to make its characteristics, including $\Gamma_{c}$, smooth. To the accuracy limits of the field measurements, $\Gamma_{c}$ is spectrally smooth at levels better than 1 part in $10^{4}$, ensuring that non-smooth features in the instrument response to the receiver noise, if any, are below the sensitivity of the observations presented here.

The total efficiency $\eta_{t}$ is estimated from a comparison of the differential antenna temperature measured as the sky drifts overhead and the expectation for this differential based on the GMOSS model for the radio sky (Sathyanarayana Rao et al. 2017a). This total efficiency, and also the reflection efficiency, are shown in Figure 2; the total efficiency represents the attenuation with which any EoR signature would be present in observed spectra. It may be noted here that the efficiency is poor and more so at lower frequencies; this was a design compromise made for SARAS 2 in that efficiency was sacrificed for spectral smoothness in the reflection efficiency and frequency independence of the beam.

\subsection{The Receiver}

The antenna signal is split coherently into two parallel paths, which are amplified separately. The splitter also adds coherent calibration noise into both paths; however, the relative phases of the sky and calibration signals in the two paths differ by $180^{\circ}$. As a consequence, the correlation spectrometer provides a difference measurement between the sky and calibration noise temperatures.

The configuration of the receiver is shown in Figure 1. A crossover switch swaps the sky and calibration signals entering the splitter. Differencing spectra recorded in the two positions of the switch cancel common-mode signals entering the two parallel signal paths. In each position of the crossover switch, the calibration noise is switched on and off to provide data for bandpass calibration. System noise couples across the parallel paths via internal reflections at the antenna and components in the receiver chains to give an additive spurious component in the measurement. This is shaped by the frequency dependence of $\Gamma_{c}$ and the relative path delay with which the direct and reflected signals arrive at the correlation spectrometer (Meys 1978).
The SARAS 2 receiver is compact, mounted directly beneath and at the antenna terminals, and the components are interconnected so as to avoid transmission lines in between. The amplified signals directly modulate lasers and transition to fibers, thus providing excellent optical isolation to the subsequent electronics located $100 \mathrm{~m}$ away. All of this helps to ensure that the additive spurious component from internal reflections and multi-path propagation of system noise is spectrally smooth; therefore, this unwanted component can also be modeled as a maximally smooth function.

\subsection{The Digital Spectrometer}

Located $100 \mathrm{~m}$ away from the antenna is a signal conditioning unit, which converts the signal back to electrical from optical and limits the band to 40-250 MHz. This is followed by a well-shielded digital spectrometer, which samples the pair of signals in the parallel paths with 10-bit precision, computes 8192-point discrete Fourier transforms, and measures the complex cross-correlation in each of 4096 frequency channels over the range $0-250 \mathrm{MHz}$. The signals are windowed in a time domain using a Blackman-Nuttall window (Nuttall 1981), which has been measured to suppress leakage of any RFI into the rest of the band by a factor of $10^{8}$.

\section{A Measurement for the $21 \mathrm{~cm}$ EoR Global Signal}

SARAS 2 was deployed at the Timbaktu Collective (latitude $=+14^{\circ} .242328, \quad$ longitude $=77^{\circ} .612606 \mathrm{E}$ ). Data were acquired over 13 nights from 2016 October to 2017 June. Ionospheric TEC for the entire observing was less than 20 units, corresponding to quiet conditions. ${ }^{5}$ Pre-processing and data calibration was performed within the MIRIAD environment (Sault et al. 1995) using custom tools.

Data were acquired cycling through each of four states: alternating the crossover switch and toggling the calibration noise in each switch position. A batch of sixteen $67.1 \mathrm{~ms}$ integrated spectra were acquired in each state of the receiver. They were Hampel filtered (Hampel 1974) to reject strong RFI and then averaged. Common-mode responses of the correlation spectrometer were rejected by differencing spectra corresponding to the two switch states; this was followed by complex bandpass calibration.

The calibrated spectra were processed using algorithms for the detection/rejection of data corrupted by lower levels of RFI. Spectra were fit with suitably high-order (10th order) Legendre polynomials over multiple overlapping bands, in order to fit out plausible models for the EoR spectrum as well as foregrounds and instrumental systematics, and outliers in the residuals were detected using median filters and rejected. This was performed in successive iterations while progressively lowering the detection threshold and repeating the fits. Data were also progressively averaged in frequency and time to detect faint RFI that may be present in contiguous channels and/or times. The algorithm was designed to avoid any asymmetric clipping of noise peaks that may result in bias in averaged residuals at levels at which the EoR signal is expected. Rejection of data corrupted by RFI resulted in useful data in the 110-200 MHz band and these calibrated spectrawithout any Legendre polynomials subtracted-were used for foreground removal and signal detection.

\footnotetext{
5 CODE data archive (ftp://ftp.unibe.ch/aiub/CODE/2016/).
} 

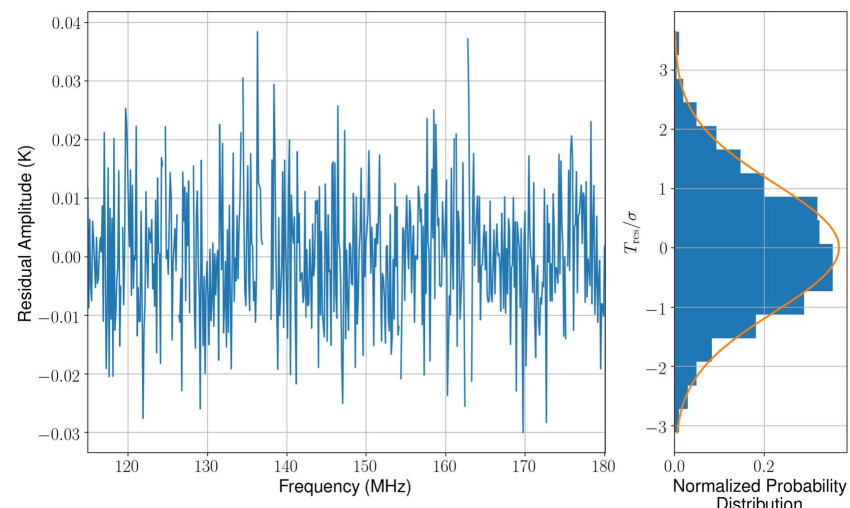

Figure 3. Residuals obtained after fitting calibrated sky data, following RFI rejection, with a 7 th order polynomial model representing the foreground. On the right is a histogram for the amplitudes along with the best-fit Gaussian. Since data rejection for RFI varies across channels, the channel amplitudes vary in their signal-to-noise ratio and, therefore, amplitudes are normalized by their $1 \sigma$ errors for the histogram.

Long-duration laboratory tests of the receiver were done with the antenna replaced by a variety of terminations: open, short, and impedance-matched terminations, and a resistor-inductorcapacitor network with $\Gamma_{c}(\nu)$ similar to that of the SARAS 2 antenna. All of these, processed as above and fitted using a single smooth function as defined in Sathyanarayana Rao et al. (2015), yielded residuals consistent with expected thermal noise.

The modeling of the foreground in the sky data was performed by fitting polynomials. This also inevitably resulted in the partial filtering out of the EoR signal. We adopted the global $21 \mathrm{~cm}$ templates predicted by the semi-numerical simulations of Cohen et al. (2016) as representative of currently allowed signals. Since these different EoR templates have different variations with frequency, we separately optimized for different templates the order of polynomial and frequency sub-band for their analysis to maximize the signal-to-noise ratio in the residual. This yields a set of residuals, individually optimized for the detection of different templates, and these are used below for deriving constraints on the EoR. These residuals have root-mean-square (rms) noise of about $11 \mathrm{mK}$ and a representative residual is shown in Figure 3.

\section{Constraints on Baryon Thermal Evolution}

Any EoR signal appearing in each of the residual spectra obtained after fitting data with appropriate polynomials would be attenuated by the total efficiency $\eta_{t}$, shown in Figure 2, and "high-pass filtered" due to the subtraction of the fitted polynomial from the data. Corresponding to any plausible EoR signal we may thus construct a "processed" EoR signal that is expected in the residual by fitting out a polynomial of the same order to the attenuated template. We have confirmed via simulations that this polynomial fitting process is linear.

To test for the presence of any plausible EoR signal in the data residual, we compute the ratio of the likelihood of the residual containing the processed signal plus expected Gaussian noise (the alternate hypothesis $H_{1}$ ), and the likelihood of the residual containing just noise (the null hypothesis $H_{0}$ ). We assume both cases to be equally likely and hence assign uniform priors. The likelihoods are defined to be

$$
P(D \mid M)=\prod_{i=1}^{N} \frac{1}{\sqrt{\left(2 \pi \sigma_{i}^{2}\right)}} e^{\frac{-\left(y_{i}-M_{i}\right)^{2}}{2 \sigma_{i}^{2}}},
$$

where $y_{i}$ is the data residual in the $i$ th frequency channel, $\sigma_{i}$ is the associated error, $M_{i}$ is the model amplitude at that channel, and $N$ is the number of independent frequency channels. We derive the measurement noise $\sigma_{i}$ by accounting for all of the data rejection for RFI, measurements of the system temperature, absolute calibration of SARAS 2, and, finally, from differences between adjacent channel data. The likelihood ratio

$$
\mathrm{LR}=\prod_{i=1}^{N} \frac{e^{\frac{-\left(y_{i}-M_{i}\right)^{2}}{2 \sigma_{i}^{2}}}}{e^{\frac{-y_{i}^{2}}{2 \sigma_{i}^{2}}}}
$$

is the ratio of likelihoods of $M$ being the processed signal to that for $M$ being zero.

To determine the significance of the likelihood ratio corresponding to any particular EoR signal template, we generate mock data sets with the same $\sigma_{i}$ distribution as that in the data residual. One data set, $D_{1}$, contains the processed EoR template plus noise, while the second data set, $D_{0}$, contains only noise. We compute likelihood ratios for $D_{0}$ and $D_{1}$ for multiple realizations of noise to derive the expected distributions of these likelihood ratios. These distributions are then used to infer the probabilities for false positives and false negatives for the likelihood ratio derived from the data depending on whether the ratio for any EoR template exceeds unity or is below unity (Kay 1998).

Given the rms noise in the data and the amplitude of the processed signal, we infer that the data is sensitive to the class of signals corresponding to late heating or poor X-ray efficiency, with $f_{X} \leqslant 0.1$ (see Cohen et al. 2016 for details), along with peak $\frac{d T_{b}}{d z} \geqslant 120 \mathrm{mK}$ per unit redshift interval corresponding to a rapid rate of reionization. We compute likelihood ratios from the residual data for the $21 \mathrm{~cm}$ templates that satisfied these criteria; there were 9 such cases out of the total of 264 in the atlas. In Figure 4 we show these templates, as well as their processed residuals.

We show in Figure 5 the likelihood ratios inferred from the data along with the expected distributions of these ratios. For almost all of the signals belonging to this class, the distributions of $D_{1}$ and $D_{0}$ are significantly separated and hence the data has the sensitivity to discriminate between the hypotheses $H_{1}$ and $H_{0}$ (presence or absence of the signal). Of these allowed signals, six are disfavored in that their likelihood ratios place them in the domain of $H_{0}$, within its 32nd to 68th percentile band, and the probability of their being false negatives is in the range $14 \%$ $28 \%$. Two signals have likelihood ratios within the 32 nd to 68 th percentile band of $H_{1}$; however, the probability that these are false alarms is as much as $25 \%-30 \%$. In the case of one signalthe one with index number 9 in the figure - the data analysis leads to a result of relatively poorer significance. The class as a whole, taking into account all nine signals, has likelihood ratios with an average probability of being false negatives of $31 \%$; therefore, the class of signals is more likely to be from $D_{0}$ than $D_{1}$. This implies that the data is more consistent with the noiseonly hypothesis than the hypothesis in which noise and template are present. We thus disfavor this class of models with $f_{X} \leqslant 0.1$, and peak $\frac{d T_{b}}{d z} \geqslant 120 \mathrm{mK}$ per unit redshift interval with $69 \%$ confidence.

The models that are disfavored by SARAS 2 all lie in the area of parameter space corresponding to late heating (Fialkov et al. 2014); actually this regime could be called "very late 
(a)

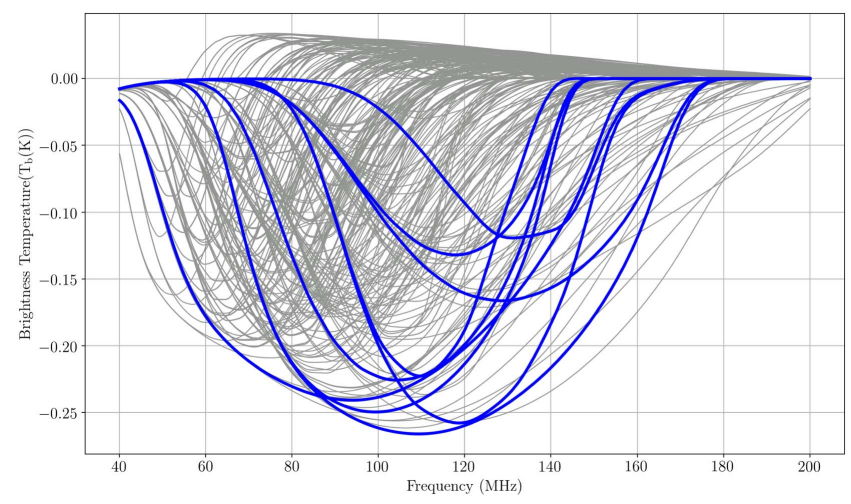

(b)

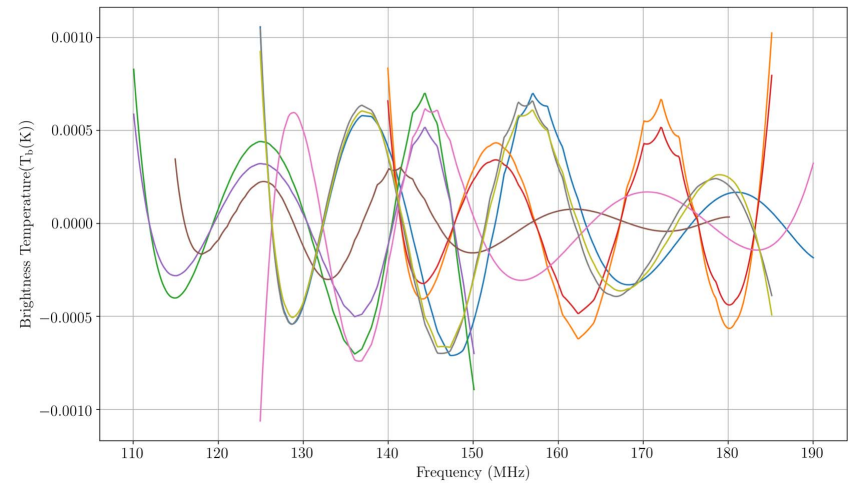

Figure 4. Panel (a) shows the atlas of $21 \mathrm{~cm}$ templates highlighting those nine that belong to the selected class of late heating and rapid reionization. The gray curves show models that are not significantly constrained by the data. Panel (b) shows the processed EoR signals, which were obtained from the templates after attenuation by the antenna efficiency followed by high-pass filtering resulting from polynomial fits.

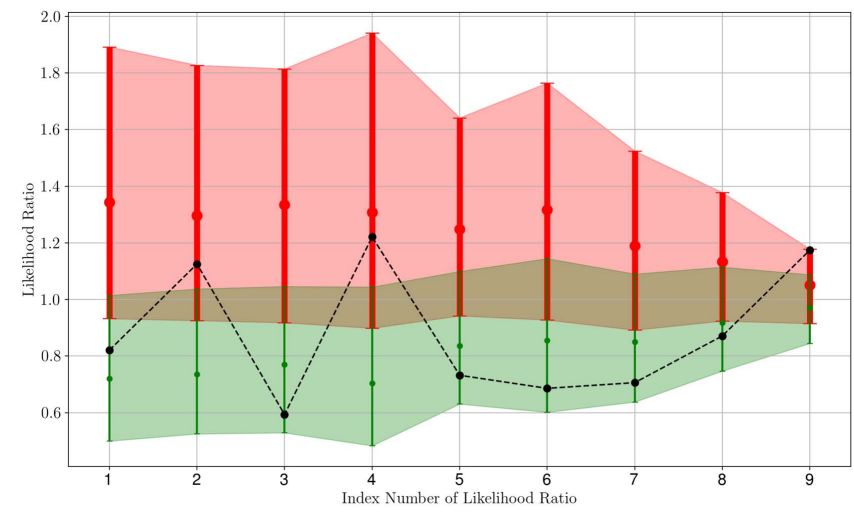

Figure 5. Likelihood ratios for the nine EoR templates that belong to the class defined in the text. For each template, we show the inferred likelihood ratio from the data (marked in black and connected using a dotted line). We also show the extents (from 32nd to 68th percentile) of the distributions of $D_{1}$ and $D_{0}$ as shaded regions. The regions corresponding to the hypotheses $H_{1}$ (upper vertical column) and $H_{0}$ (lower vertical column) are shown in red and green, respectively; their medians are shown using filled circles.

heating," in which cosmic reionization ends without the global $21 \mathrm{~cm}$ signal having reached emission. More specifically, SARAS 2 disfavors models that have late (i.e., weak) X-ray heating and a rapid end to reionization (due, for example, to large galaxies dominating star formation and a large mean free path available within the ionized bubbles).
In summary, we disfavor the class of global $21 \mathrm{~cm}$ models that represent late heating or poor X-ray efficiency, with $f_{X} \leqslant 0.1$, and with peak $\frac{d T_{b}}{d z} \geqslant 120 \mathrm{mK}$ per unit redshift interval corresponding to a rapid rate of reionization with $69 \%$ confidence. These results are intriguing and we are devising better strategies for foreground modeling toward minimizing loss in signal amplitudes.

We thank the anonymous referee for valuable comments that led to improved methods of data analysis. We thank the staff at Gauribidanur Field Station for assistance with system tests and measurements, and the Mechanical and Electronics Engineering Groups at Raman Research Institute for building and assembling SARAS 2. Santosh Harish and Divya Jayasankar implemented real-time software and monitoring. Logistics and technical support for observations was provided by Indian Astronomical Observatory, Leh operated by Indian Institute of Astrophysics, and Timbaktu Collective, India. For R.B. and A. C. this project/publication was made possible through the support of a grant from the John Templeton Foundation. The opinions expressed in this publication are those of the author(s) and do not necessarily reflect the views of the John Templeton Foundation.

\section{ORCID iDs}

Saurabh Singh (10) https://orcid.org/0000-0001-7755-902X Ravi Subrahmanyan (10 https://orcid.org/0000-0001-9913-900X N. Udaya Shankar (10 https://orcid.org/0000-0001-8974-3369 Mayuri Sathyanarayana Rao (10 https://orcid.org/0000-00029761-3676

Anastasia Fialkov (D) https://orcid.org/0000-0002-1369-633X

A. Raghunathan (1D https://orcid.org/0000-0003-1929-9869

\section{References}

Barkana, R. 2016, PhR, 645, 1

Barkana, R., \& Loeb, A. 2001, PhR, 349, 125

Beardsley, A. P., Hazelton, B. J., Sullivan, I. S., et al. 2016, ApJ, 833, 102

Bernardi, G., McQuinn, M., \& Greenhill, L. J. 2015, ApJ, 799, 90

Bernardi, G., Zwart, J. T. L., Price, D., et al. 2016, MNRAS, 461, 2847

Bowman, J. D., \& Rogers, A. E. E. 2010, Natur, 468, 796

Ciardi, B., \& Ferrara, A. 2005, SSRv, 116, 625

Cohen, A., Fialkov, A., Barkana, R., \& Lotem, M. 2016, MNRAS, submitted (arXiv:1609.02312)

Fan, X., Carilli, C. L., \& Keating, B. 2006, ARA\&A, 44, 415

Fialkov, A., Barkana, R., \& Visbal, E. 2014, Natur, 506, 197

Field, G. B. 1958, PIRE, 46, 240

Furlanetto, S. R. 2006, MNRAS, 371, 867

Furlanetto, S. R., Oh, S. P., \& Briggs, F. H. 2006, PhR, 433, 181

Haiman, Z. 2016, Cosmic Reionization and the First Nonlinear Structures in the Universe (Cham: Springer International), 1

Hampel, F. R. 1974, J. Am. Stat. Assoc., 69, 383

Harker, G. J. A. 2015, MNRAS, 449, L21

Harker, G. J. A., Mirocha, J., Burns, J. O., \& Pritchard, J. R. 2016, MNRAS, 455,3829

Hinshaw, G., Larson, D., Komatsu, E., et al. 2013, ApJS, 208, 19

Kay, S. M. 1998, Fundamentals of Statistical Signal Processing, Vol. II: Detection Theory (Englewood Cliffs, NJ: Prentice-Hall)

Liu, A., Pritchard, J. R., Tegmark, M., \& Loeb, A. 2013, PhRvD, 87, 043002

Liu, A., \& Tegmark, M. 2012, MNRAS, 419, 3491

Madau, P., \& Fragos, T. 2017, ApJ, 840, 39

Madau, P., Meiksin, A., \& Rees, M. J. 1997, ApJ, 475, 429

McGreer, I. D., Mesinger, A., \& D’Odorico, V. 2015, MNRAS, 447, 499

McQuinn, M. 2016, ARA\&A, 54, 313

Meys, R. 1978, ITMTT, 26, 34

Mirocha, J., Furlanetto, S. R., \& Sun, G. 2017, MNRAS, 464, 1365

Mirocha, J., Harker, G. J. A., \& Burns, J. O. 2013, ApJ, 777, 118 
Monsalve, R. A., Rogers, A. E. E., Bowman, J. D., \& Mozdzen, T. J. 2017, ApJ, 835, 49

Morandi, A., \& Barkana, R. 2012, MNRAS, 424, 2551

Nuttall, A. H. 1981, ITASS, 29, 84

Patil, A. H., Yatawatta, S., Koopmans, L. V. E., et al. 2017, ApJ, 838, 65

Patra, N., Subrahmanyan, R., Raghunathan, A., \& Udaya Shankar, N. 2013, ExA, 36, 319

Planck Collaboration, Adam, R., Aghanim, N., et al. 2016, A\&A, 596, A108

Pober, J. C., Ali, Z. S., Parsons, A. R., et al. 2015, ApJ, 809, 62

Pritchard, J. R., \& Loeb, A. 2012, RPPh, 75, 086901

Sathyanarayana Rao, M., Subrahmanyan, R., Udaya Shankar, N., \& Chluba, J. 2015, ApJ, 810, 3

Sathyanarayana Rao, M., Subrahmanyan, R., Udaya Shankar, N., \& Chluba, J. 2017a, AJ, 153, 26

Sathyanarayana Rao, M., Subrahmanyan, R., Udaya Shankar, N., \& Chluba, J. 2017b, ApJ, 840, 33
Sault, R. J., Teuben, P. J., \& Wright, M. C. H. 1995, in ASP Conf. Ser. 77, Astronomical Data Analysis Software and Systems IV, ed. R. A. Shaw, H. E. Payne, \& J. J. E. Hayes (San Francisco, CA: ASP), 433

Shaver, P. A., Windhorst, R. A., Madau, P., \& de Bruyn, A. G. 1999, A\&A, 345,380

Sokolowski, M., Tremblay, S. E., Wayth, R. B., et al. 2015a, PASA, 32, e004

Sokolowski, M., Wayth, R. B., Tremblay, S. E., et al. 2015b, ApJ, 813, 18

Vedantham, H. K., Koopmans, L. V. E., de Bruyn, A. G., et al. 2014, MNRAS, 437, 1056

Voytek, T. C., Natarajan, A., Jáuregui García, J. M., Peterson, J. B., \& López-Cruz, O. 2014, ApJL, 782, L9

Wouthuysen, S. A. 1952, AJ, 57, 31

Zaroubi, S. 2013, in The First Galaxies, Vol. 396, ed. T. Wiklind, B. Mobasher, \& V. Bromm (Berlin: Springer), 45

Zheng, Z.-Y., Wang, J., Rhoads, J., et al. 2017, ApJL, 842, L22 